\title{
The Crumbs3 Polarity Protein
}

\author{
Ben Margolis \\ Department of Internal Medicine, University of Michigan Medical School, Ann Arbor, Michigan 48109-5680 \\ Correspondence: bmargoli@umich.edu
}

The Crumbs proteins are evolutionarily conserved apical transmembrane proteins. Drosophila Crumbs was discovered via its crucial role in epithelial polarity during fly embryogenesis. Crumbs proteins have variable extracellular domains but a highly conserved intracellular domain that can bind FERM and PDZ domain proteins. Mammals have three Crumbs genes and this review focuses on Crumbs3, the major Crumbs isoform expressed in mammalian epithelial cells. Although initial work has highlighted the role of Crumbs3 in polarity, more recent studies have found it has an important role in tissue morphogenesis functioning as a linker between the apical membrane and the actin cytoskeleton. In addition, recent publications have linked Crumbs3 to growth control via regulation of the Hippo/ Yap pathway.

Epithelia polarize into apical and basolateral Emembranes with unique protein compositions. This polarization is crucial for proper epithelial functions such as ion transport and barrier protection. In mammalian epithelia, a seal known as the tight junction separates the apical and basolateral membranes (Shin et al. 2006; Nelson 2009). The tight junction is composed of transmembrane proteins, primarily claudins that are organized by scaffold proteins such as the zonula occludens. Another junction important for polarity is the adherens junction, which sits on the lateral membrane basal to the tight junction. Its primary constituents are the cadherins. Adherens junctions are mediators of cell-cell adhesion, a component of polarity in epithelial sheets and tubes (Nelson et al. 2013).

Work over the last 20 years has indicated that apicobasal polarity is initiated and maintained by mutual antagonism of apical and basolateral protein complexes. In lower organisms, such as Drosophila, polarity is maintained solely by antagonistic polarity complexes. In mammalian cells, this biochemical polarization is further enforced by the tight junction seal (Shin et al. 2006). There are two main evolutionarily conserved apical polarity complexes: the Crumbs complex and the partitioning defective (Par) complex (Pieczynski and Margolis 2011). The Crumbs complex consists of three major proteins, Crumbs, protein associated with lin-7 one (Pals1; Stardust in Drosophila), and PALS1 associated tight junction protein (Patj), all of which are highly conserved from invertebrates to vertebrates. A similarly evolutionarily conserved polarity complex is the Par complex that consists of Par3, Par6, and atypical protein kinase C (aPKC) (Pieczynski and Margolis 2011). aPKC is a crucial component of the apical

Editor: Keith E. Mostov

Additional Perspectives on Cell Polarity available at www.cshperspectives.org

Copyright (C) 2018 Cold Spring Harbor Laboratory Press; all rights reserved; doi: 10.1101/cshperspect.a027961

Cite this article as Cold Spring Harb Perspect Biol 2018;10:a027961 
B. Margolis

polarity complex as phosphorylation is felt to be the main biochemical effector mechanism that results in membrane polarization. This review will focus on the Crumbs complex and in particular the Crumbs3 protein, which is the major Crumbs isoform found in mammalian epithelia. Although Crumbs was initially identified as a polarity protein, recent studies have shown that it has multiple functions in epithelial cells.

\section{THE CRUMBS PROTEINS}

Crumbs was first identified in Drosophila as a regulator of epithelial polarity. In Drosophila development, Crumbs is crucial for polarization of the ectoderm that is formed after gastrulation. The external cuticle, which is derived from this ectoderm, is disrupted when Crumbs is not present leading to a speckled (crumbslike) appearance (Tepass et al. 1990). The Drosophila Crumbs protein has a large extracellular domain with 30 epidermal growth factor (EGF)-like repeats, a single-span transmembrane region and a conserved cytoplasmic carboxyl terminus. The intracellular carboxyl terminus contains a FERM ( $\mathrm{F}$ for 4.1 protein, E for ezrin, $\mathrm{R}$ for radixin, and $\mathrm{M}$ for moesin)binding domain and a PDZ (P for postsynaptic density-95, D for discs large, and $\mathrm{Z}$ for zonula occludens1)-binding domain. In vertebrates, there are three Crumbs genes termed Crumbs1, Crumbs2, and Crumbs3. Crumbs1 and Crumbs2 have large extracellular domains like the Drosophila Crumbs, whereas Crumbs3 has a very short extracellular domain (Makarova et al. 2003). Crumbs1 is primarily localized to the retinal pigmented epithelium of the eye and mutations in the gene have been linked to retinal dystrophies (Alves et al. 2014). Many of these mutations are found in the extracellular domain of Crumbs1, which is consistent with the finding that mutations in the extracellular domain of Drosophila Crumbs leads to abnormal photoreceptor morphogenesis in flies (Alves et al. 2014). Knockout of Crumbs1 in mice leads to photoreceptor degeneration after 9 months of age (van de Pavert et al. 2004). Crumbs 2 gene expression has been detected in brain, testis, uterus, eye, and embryonic tissue (van den Hurk et al. 2005). Knockout of Crumbs 2 in mice results in embryonic lethality at the late gastrulation stage (Xiao et al. 2011). Recent work has described patients with Crumbs 2 mutations resulting in ventriculomegaly and nephrotic syndrome (Ebarasi et al. 2015; Slavotinek et al. 2015).

The Crumbs 3 gene is expressed in the majority of mammalian epithelia (Makarova et al. 2003). Crumbs3 exists in two variants as a result of alternate splicing of the final exon of the Crumbs 3 gene. This alternate splicing results in a 22-amino acid difference in the carboxyl terminus, altering the sequence of the PDZbinding domain (Fan et al. 2007). Crumbs3a has the carboxy-terminal sequence glutamic acid-arginine-leucine-isoleucine (ERLI) amino acids, which comprise its PDZ-binding motif. Crumbs3b has a different carboxyl terminus, ending in the sequence cysteine-leucineproline-isoleucine (CLPI) that does not bind PDZ domains. The mechanisms regulating the abundance of each splice variant are not yet determined. The function of the Crumbs3 protein is discussed extensively below.

\section{CRUMBS-BINDING PARTNERS}

Crumbs binds to the conserved polarity proteins Pals1 (Drosophila Stardust) and Par6 via its PDZ-binding carboxy-terminal sequence, ERLI (Fig. 1) (Hurd et al. 2003; Lemmers et al. 2004). Pals1/Stardust is a membraneassociated guanylate kinase (MAGUK) protein with the core complex of PDZ, Src homology domain 3 (SH3), and guanylate kinase domain (GUK) (Kamberov et al. 2000; Funke et al. 2005). Loss-of-function of Pals1/Stardust in Drosophila closely phenocopies loss of Crumbs (Bachmann et al. 2001; Hong et al. 2001). The complex of Crumbs3 bound to Pals1 has been studied at a crystallographic level. Although the Crumbs3 ERLI carboxy-terminal sequence is a classic PDZ-binding motif, these structural studies show that the PDZ, SH3, and GUK domains are all required for the high-affinity interaction between Crumbs3 and Pals1 (Li et al. 2014). The binding of Pals1 to Crumbs appears 
The Crumbs3 Polarity Protein

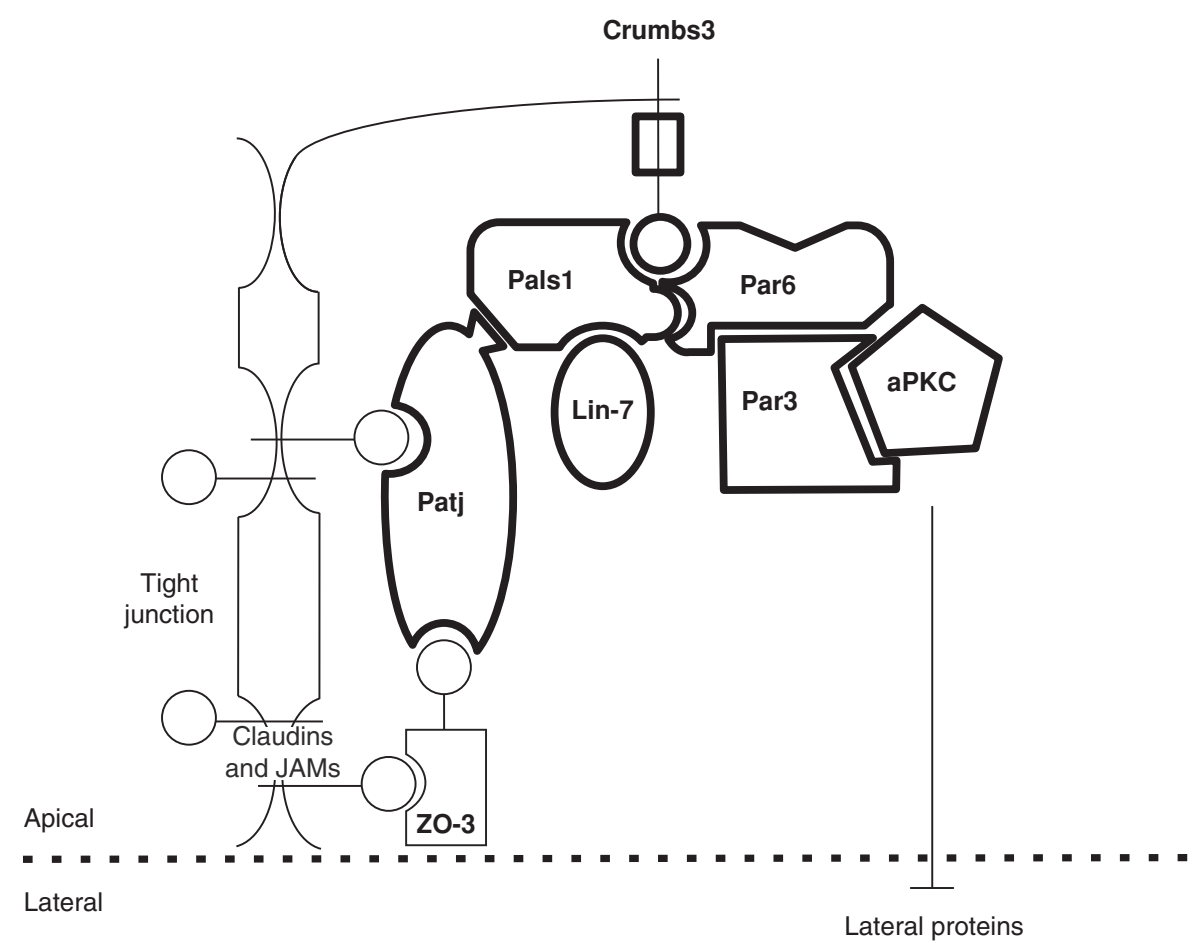

Figure 1. Crumbs3 PDZ domain interactions. Through its carboxy-terminal tail (circle), Crumbs3 can bind two PDZ domain proteins, Pals1 (protein associated with lin-7 one) and Par6. Par6 can bind Par3 as well as atypical protein kinase $\mathrm{C}$ ( $\mathrm{aPKC}$ ), and aPKC can regulate basolateral polarity complexes via phosphorylation. Pals1 binds Patj (PALS1 associated tight junction protein) and Lin-7 via L27 domains. Patj has 10 PDZ domains that can mediate numerous interactions including to transmembrane tight junction proteins such as claudins and junctional adhesion molecules (JAMs), as well as to tight junction scaffolding proteins such as zonula occludens 3 (ZO-3).

to be of a much higher affinity than the binding of Crumbs to Par6 (Whitney et al. 2016). At its amino terminus, Pals1 has two L27 domains (Doerks et al. 2000). One of these L27 domains binds to the small PDZ scaffold protein Lin-7 (Kamberov et al. 2000). The other L27 domains bind the multi-PDZ domain proteins, Patj and multiple PDZ domain protein 1 (Mupp1) (Roh et al. 2002; Assemat et al. 2013). Patj has multiple PDZ domains and can connect the Crumbs3 complex to proteins of the tight junction such as the zonula occludens proteins (Adachi et al. 2009). The nature and extent of the interaction of the Par complex with the Crumbs complex varies depending on the epithelia in which it is studied. There are multiple regulatory factors that control these interactions, including aPKC as well as small $\mathrm{G}$ proteins such as $\mathrm{Cdc} 42$ (Pieczynski and Margolis 2011; Tepass 2012; Whitney et al. 2016).

Several proteins have also been identified that bind to the Crumbs FERM-binding domain (Fig. 2). Yurt was shown to bind the FERM-binding domain of Drosophila Crumbs and function as a negative regulator of Crumbs (Laprise et al. 2006). Studies have also indicated that the mammalian orthologs of Yurt, YMO1, EHM2, and EPB41L5 have a similar function in mammalian cells and form a novel complex with Crumbs3 and Pals1 (Laprise et al. 2006, 2009; Gosens et al. 2007). In Drosophila Crumbs, the FERM-binding domain interacts with the expanded protein to regulate growth control (see below) (Grzeschik et al. 2010; Ling 
B. Margolis

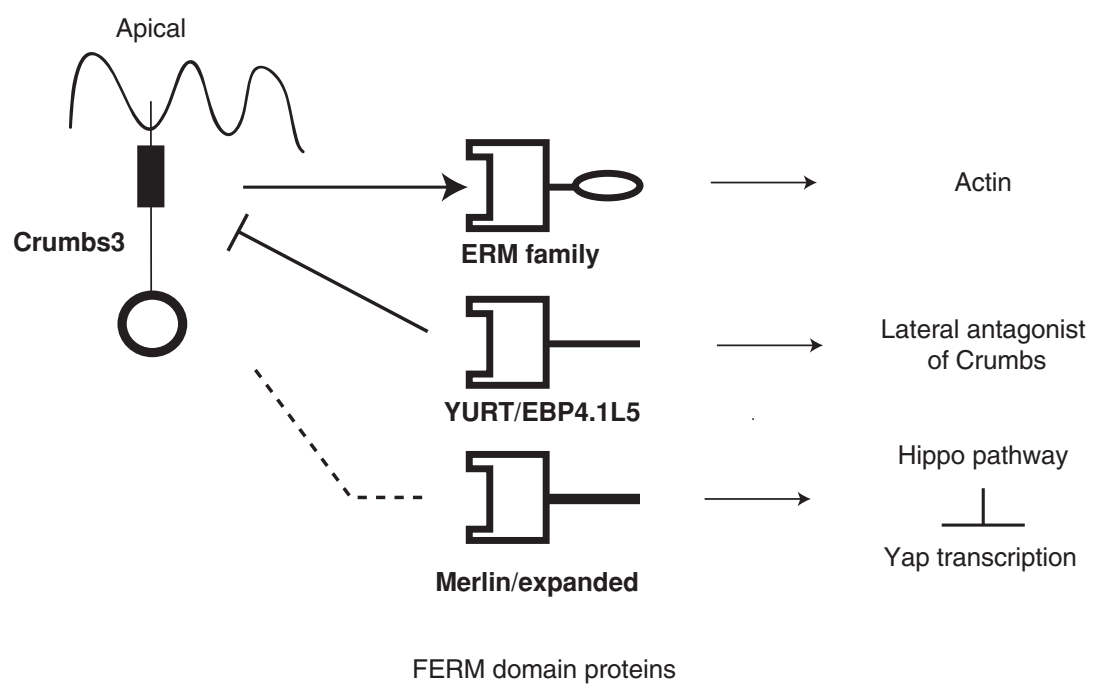

Figure 2. FERM-binding partners of Crumbs3. Through its FERM-binding domain (rectangle), Crumbs3 can connect to FERM domain-containing proteins. Crumbs3 can connect the apical membrane to the actin cytoskeleton and regulate the cell cortex via ezrin-radixin-moesin (ERM) proteins. Crumbs3 also binds Yurt family proteins, which negatively regulate Crumbs polarity signaling. Crumbs3 interacts with FERM domain proteins that regulate the Hippo/Yap pathway to control cell proliferation.

et al. 2010; Robinson et al. 2010). The third family of proteins that bind to the FERM domain are members of the ezrin-radixin-moesin (ERM) family of proteins (Medina et al. 2002). ERM proteins link the cell membrane to the underlying actin cytoskeleton and serve as scaffolds for cell signaling (Fehon et al. 2010; Ponuwei 2016). Recently, a structure has been published of Crumbs 3 binding to moesin. The structure shows that when Crumbs 3 binds to ERM proteins via its FERM-binding motif, it loses its ability to bind PDZ partners such as Pals1 (Wei et al. 2015). The Crumbs3b isoform only has the FERM-binding domain and thus may have unique functions (Fan et al. 2007; Tilston-Lunel et al. 2016).

\section{FUNCTIONS OF THE CRUMBS3 PROTEIN}

\section{Control of Polarity}

The role of Crumbs 3 in epithelial polarity has been extensively studied. Overexpression of Crumbs3 leads to an expansion of the apical surface and defects in tight junctions (Roh et al. 2003; Lemmers et al. 2004). Knockdown of Crumbs 3 in Madin-Darby canine kidney (MDCK) cells altered cilia generation but did not affect polarity or tight junctions when cells were grown in monolayer culture (Fan et al. 2004). In contrast, restoration of Crumbs 3 in MCF10a breast epithelial cells promoted tight junction formation (Fogg et al. 2005). More marked effects of Crumbs3 knockdown were seen when cells were grown in three-dimensional culture systems. MDCK cells form a sphere of cells with a perfect lumen in three-dimensional culture but this is impaired in cells where Crumbs3 is knocked down (Torkko et al. 2008; Schluter et al. 2009).

However, surprisingly our group and others were not able to show an obvious defect in polarity or cell-cell junctions in Crumbs 3 knockout mice (Whiteman et al. 2014; Charrier et al. 2015). Loss of Crumbs 3 did lead to a significant decrease in the expression of Pals1 and Patj. However, despite losing almost all the proteins of the Crumbs complex, tight junctions appeared normal. Lumen-containing structures such as the tubule of the kidney and bronchiole 
The Crumbs3 Polarity Protein

of the lung were able to develop (Whiteman et al. 2014; Charrier et al. 2015). This was in marked contrast to what was seen in Crumbs3 knockdown cells in three-dimensional culture (Torkko et al. 2008; Schluter et al. 2009). However, it should be noted that Crumbs3 knockout mice die at birth as a result of lung defects. The cause of this death is not clear but increased glycosylated material in bronchial lumens and alveoli appeared to have blocked the initiation of breathing. In the kidney, we found that the vast majority of tubules formed normally but we did detect a small number of cysts. These cysts could be related to polarity defects or loss of cilia in a small subset of cells, although in most renal cells cilia were detected (Whiteman et al. 2014).

There may be several reasons why the polarity defects were not more marked in the Crumbs 3 knockout mice. The simplest explanation is that Crumbs 3 is not primarily involved in polarity regulation. As discussed below, its main effects may be primarily related to actomyosin dynamics or growth control. A second explanation is that there is redundancy in polarity signaling. One form of redundancy would come in the form of Crumbs 2 expression. Knockout of Crumbs 2 in mice leads to embryonic death at gastrulation very similar to what is seen with loss of Drosophila Crumbs (Xiao et al. 2011). However, we did not detect Crumbs 1 or Crumbs 2 overexpression in tissues that had lost Crumbs3 (Whiteman et al. 2014).

It seems that the most likely explanation is that other polarity proteins in particular, the Par proteins, can initiate or maintain polarity in the absence of the Crumbs complex. For example, there are no polarity defects in the absence of Crumbs early in the development of the renal tubule of the fly. However, Drosophila Par3 also known as Bazooka is essential for polarity at this stage (Campbell et al. 2009). In fact, there are several tissues in Drosophila in which Crumbs is not essential for polarity possibly because of redundancy with other polarity proteins (Tepass 2012). However, just like Drosophila, there are likely to be a fraction of cells/ tissues in mammals in which Crumbs3 is required for polarity (Szymaniak et al. 2015).

\section{MEMBRANE CYTOSKELETAL INTERACTIONS AND TISSUE MORPHOGENESIS}

If Crumbs3 is not essential for polarity what might be its function in epithelia and other cells? Again, studies in Drosophila point to these other functions. In Drosophila, the trachea originates from ten small groupings (placodes) of epithelial cells. The placodes then invaginate because of apical constriction to form a sac that matures into tube-like structures. These tubes fuse to form the trachea and respiratory system. Crumbs aggregates at the dorsal surface that is the site of apical constriction. Crumbs controls this apical constriction via its FERMbinding domain and its binding partner, moesin (Letizia et al. 2011; Klose et al. 2013). The Crumbs/moesin interaction likely ties the apical membrane to the underlying cytoskeleton to promote apical constriction and cellular movement. Similar roles for Crumbs and its binding partner moesin have been found in the actomyosin contraction essential for cell movement required for dorsal closure of the Drosophila embryo (Flores-Benitez and Knust 2015).

These results in Drosophila foreshadowed the defects in the Crumbs3 knockout mice. In addition to the pulmonary defects seen in these knockout mice, another major phenotype was abnormal intestinal development (Whiteman et al. 2014; Charrier et al. 2015). In the mouse, the small intestine is a tube-shaped structure at E14.5, but by E15.5 morphogenic movements have occurred leading to a villus structure (Walton et al. 2016). These villi are finger-like projections that increase the surface area of the intestine to allow for increased absorption of nutrients. Further increase in surface area is mediated by microvilli that coat the apical surface of the villi. The villi should be present as individual projections into the lumen but in Crumbs 3 knockout mice these villi are fused. In addition, the intestinal microvilli are short and disorganized. These intestinal defects closely phenocopy ezrin knockout mice that also display villus fusion and shortened microvilli (Saotome et al. 2004; Casaletto et al. 2011). As ezrin is a member of the ERM family of proteins 
B. Margolis

it binds to Crumbs3 via the FERM domain (Whiteman et al. 2014; Tilston-Lunel et al. 2016). Thus, there are likely parallels between Crumbs and moesin control of morphogenesis in Drosophila and Crumbs3 and ezrin control of mammalian intestinal development. In the Crumbs3 knockout mice, we detected membrane blebbing another phenotype consistent with loss of Crumbs interaction with ERM proteins (Charras et al. 2006; Casaletto et al. 2011). Additional studies have highlighted a role for Crumbs 3 in the control of the actin cytoskeleton. In testes, it was found that Crumbs 3 could interact with the Arp2/3 complex that regulates actin branching (Gao et al. 2016). In another study, it was shown that Crumbs3 through EHM2 (a homolog of Drosophila yurt) can promote Rho activation and actomyosin reorganization (Loie et al. 2015). Taken together, these results indicate an important role for Crumbs3 in the control of the actin cytoskeleton. It can be argued that, in the Crumbs 3 knockout mice, the predominant phenotype is the defect in the linkage between the apical membrane and the actomyosin cytoskeleton.

\section{GROWTH CONTROL}

Another potential function for Crumbs 3 is the regulation of growth control. In Drosophila, it is known that increased or decreased Crumbs expression leads to cellular overgrowth (Sotillos et al. 2004; Lu and Bilder 2005; Chen et al. 2010; Ling et al. 2010). Initial studies attributed this to loss of polarity and possible defects in localization of growth factors or their receptors (Hariharan and Bilder 2006). However, more recent studies have indicated that the aberrant growth induced by loss of Crumbs is caused by dysregulation of the Hippo pathway. The Hippo pathway is known to be an important regulator of cell growth (Elbediwy et al. 2016; Sun and Irvine 2016). In Drosophila, the Hippo and Warts kinases regulate the transcription coactivator Yorkie. When Yorkie is in the nucleus, it activates cell proliferation. Hippo and Warts function to phosphorylate Yorkie and exclude it from the nucleus. The Warts kinase is activated through phosphorylation by the Hippo ki- nase and Hippo and Warts kinases are brought together by several linker proteins that can be controlled by upstream signaling. The Drosophila Crumbs protein can control the activity of the Hippo and Warts kinases through linker proteins such as the FERM domain protein, Expanded (Robinson et al. 2010; Sun and Irvine 2016). When Crumbs is at the apical membrane, acting as a marker of a fully polarized cell, it can bind Expanded, activate the Hippo pathway, and block Yorkie nuclear action. The role of Crumbs in cell growth has been best studied in the Drosophila wing (Chen et al. 2010; Ling et al. 2010; Robinson et al. 2010). In the Drosophila wing, loss of Crumbs does not alter polarity because of redundancy with other polarity proteins such as Par3. However, loss of Crumbs leads to loss of Hippo signaling and maintenance of Yorkie in the nucleus leading to hyperproliferation.

Mammalian cells have two Warts homologs, Lats1 and Lats2, two Hippo homologs, Mst1 and Mst2, and two Yorkie homologs, Yap and Taz. The Expanded homologs have been more difficult to identify and this role may be served by multiple proteins, including merlin/neurofibromatosis2 and the angiomotins (Sun and Irvine 2016). In mammalian cells, there are more limited studies showing the connection between Crumbs genes and cellular overgrowth. A group of studies from Varelas and coworkers, however, have pointed to a connection with Crumbs3. Their initial work showed that the Crumbs3 complex interacts with Yap and Taz (Varelas et al. 2010). They found that Yorkie localizes to the cytoplasm in confluent mouse mammary epithelial cells. Knockdown of Crumbs3 in confluent cells led to the movement of Yap to the nucleus. This indicates that Crumbs 3 may play an important role in epithelial proliferation similar to what is seen with Crumbs in Drosophila. In more recent work, these investigators have looked at the lung abnormalities in Crumbs 3 knockout mice (Szymaniak et al. 2015). They focused on the cells that lead to the development of the mouse lung proximal airway. In these cells, it was found that loss of Crumbs 3 leads to abnormal activation of the Yap pathway. In addition, there were differentiation and polarity de- 
fects in the proximal airways. These defects may have been responsible for the abnormal development of the lungs and death of the Crumbs3 mice. It is important to note that the Crumbs 3 knockout mice do not have a global problem with growth control (Whiteman et al. 2014). In general, the organs are not larger and these defects in Hippo/Yap signaling are not seen globally in the mouse. However, as in Drosophila, it is likely that Crumbs3 has different cellular functions depending on the cell type and context.

Finding that loss of Crumbs3 leads to increased Yap signaling in the nucleus could have important implications for human cancer. Enhanced Yap signaling has been found in many cancers and correlates with a worse prognosis (Zanconato et al. 2016). In cancer, Crumbs3 is down-regulated in the process of epithelial to mesenchymal transition. This transition is induced by transcription factors such as Snail and Zeb1 that have direct effects on the Crumbs3 promoter (Aigner et al. 2007; Whiteman et al. 2008). In a mouse model, down-regulation of Crumbs 3 leads to enhanced proliferation and metastasis (Karp et al. 2008), and recent work shows that down-regulation of Crumbs 3 may lead to a worse prognosis in human cancer (Mao et al. 2015).

\section{CONCLUDING REMARKS}

In summary, we have a good understanding of Crumbs 3 targeting and binding partners. However, we are still trying to understand its role in vivo in mammalian tissues. Findings to date indicate that Crumbs3 controls several physiologic processes, including cell polarity, actin dynamics, and cell growth. There is likely cross talk between these Crumbs pathways. For example, actin dynamics may regulate the Hippo pathway (Elbediwy et al. 2016). In addition, members of the Yurt family may antagonize Crumbs polarity signaling while enhancing Crumbs regulation of the actin cytoskeleton (Loie et al. 2015). Crumbs does not appear to have all these effects in all cells; rather, there are subsets of cells that require Crumbs 3 for these various actions. In addition, redundancy with other Crumbs genes and other polarity proteins such as Par3 make the interpretation more complex. Finally, the physiologic role of Crumbs 3 may only be revealed when animals are stressed with environmental changes or disease. Additional studies with tissue-specific knockouts of Crumbs3 will be revealing in this regard.

\section{REFERENCES}

Adachi M, Hamazaki Y, Kobayashi Y, Itoh M, Tsukita S, Furuse M. 2009. Similar and distinct properties of MUPP1 and Patj, two homologous PDZ domain-containing tight-junction proteins. Mol Cell Biol 29: 23722389.

Aigner K, Dampier B, Descovich L, Mikula M, Sultan A, Schreiber M, Mikulits W, Brabletz T, Strand D, Obrist P, et al. 2007. The transcription factor ZEB1 ( $\delta E F 1)$ promotes tumour cell dedifferentiation by repressing master regulators of epithelial polarity. Oncogene 26: 69796988.

Alves CH, Pellissier LP, Wijnholds J. 2014. The CRB1 and adherens junction complex proteins in retinal development and maintenance. Prog Retin Eye Res 40: 35-52.

Assemat E, Crost E, Ponserre M, Wijnholds J, Le Bivic A, Massey-Harroche D. 2013. The multi-PDZ domain protein-1 (MUPP-1) expression regulates cellular levels of the PALS-1/PATJ polarity complex. Exp Cell Res 319: 2514-2525.

Bachmann A, Schneider M, Theilenberg E, Grawe F, Knust E. 2001. Drosophila Stardust is a partner of Crumbs in the control of epithelial cell polarity. Nature 414: 638-643.

Campbell K, Knust E, Skaer H. 2009. Crumbs stabilises epithelial polarity during tissue remodelling. J Cell Sci 122: $2604-2612$.

Casaletto JB, Saotome I, Curto M, McClatchey AI. 2011. Ezrin-mediated apical integrity is required for intestinal homeostasis. Proc Natl Acad Sci 108: 11924-11929.

Charras GT, Hu CK, Coughlin M, Mitchison TJ. 2006. Reassembly of contractile actin cortex in cell blebs. J Cell Biol 175: $477-490$.

Charrier LE, Loie E, Laprise P. 2015. Mouse Crumbs3 sustains epithelial tissue morphogenesis in vivo. Sci Rep 5: 17699.

Chen CL, Gajewski KM, Hamaratoglu F, Bossuyt W, Sansores-Garcia L, Tao C, Halder G. 2010. The apicalbasal cell polarity determinant Crumbs regulates Hippo signaling in Drosophila. Proc Natl Acad Sci 107: 1581015815.

Doerks T, Bork P, Kamberov E, Makarova O, Muecke S, Margolis B. 2000. L27, a novel heterodimerization domain in receptor targeting proteins Lin-2 and Lin-7. Trends Biochem Sci 25: 317-318.

Ebarasi L, Ashraf S, Bierzynska A, Gee HY, McCarthy HJ, Lovric S, Sadowski CE, Pabst W, Vega-Warner V, Fang H, et al. 2015. Defects of CRB2 cause steroid-resistant nephrotic syndrome. Am J Hum Genet 96: 153-161. 
B. Margolis

Elbediwy A, Vincent-Mistiaen ZI, Thompson BJ. 2016. YAP and TAZ in epithelial stem cells: A sensor for cell polarity, mechanical forces and tissue damage. BioEssays 38: 644653.

Fan S, Hurd TW, Liu CJ, Straight SW, Weimbs T, Hurd EA, Domino SE, Margolis B. 2004. Polarity proteins control ciliogenesis via kinesin motor interactions. Curr Biol 14: 1451-1461.

Fan S, Fogg V, Wang Q, Chen XW, Liu CJ, Margolis B. 2007. A novel Crumbs3 isoform regulates cell division and ciliogenesis via importin $\beta$ interactions. J Cell Biol 178: 387-398.

Fehon RG, McClatchey AI, Bretscher A. 2010. Organizing the cell cortex: The role of ERM proteins. Nat Rev Mol Cell Biol 11: 276-287.

Flores-Benitez D, Knust E. 2015. Crumbs is an essential regulator of cytoskeletal dynamics and cell-cell adhesion during dorsal closure in Drosophila. eLife 4: e07398.

Fogg VC, Liu CJ, Margolis B. 2005. Multiple regions of Crumbs 3 are required for tight junction formation in MCF10A cells. J Cell Sci 118: 2859-2869.

Funke L, Dakoji S, Bredt DS. 2005. Membrane-associated guanylate kinases regulate adhesion and plasticity at cell junctions. Annu Rev Biochem 74: 219-245.

Gao Y, Lui WY, Lee WM, Cheng CY. 2016. Polarity protein Crumbs homolog-3 (CRB3) regulates ectoplasmic specialization dynamics through its action on F-actin organization in Sertoli cells. Sci Rep 6: 28589.

Gosens I, Sessa A, den Hollander AI, Letteboer SJ, Belloni V, Arends ML, Le Bivic A, Cremers FP, Broccoli V, Roepman R. 2007. FERM protein EPB41L5 is a novel member of the mammalian CRB-MPP5 polarity complex. Exp Cell Res 313: 3959-3970.

Grzeschik N, Parsons L, Allott M, Harvey K, Richardson H 2010. Lgl, aPKC, and Crumbs regulate the Salvador/ Warts/Hippo pathway through two distinct mechanisms. Curr Biol 20: 573-581.

Hariharan IK, Bilder D. 2006. Regulation of imaginal disc growth by tumor-suppressor genes in Drosophila. Annu Rev Genet 40: 335-361.

Hong Y, Stronach B, Perrimon N, Jan LY, Jan YN. 2001. Drosophila Stardust interacts with Crumbs to control polarity of epithelia but not neuroblasts. Nature 414: 634638.

Hurd TW, Gao L, Roh MH, Macara IG, Margolis B. 2003. Direct interaction of two polarity complexes implicated in epithelial tight junction assembly. Nat Cell Biol 5: 137142 .

Kamberov E, Makarova O, Roh M, Liu A, Karnak D, Straight S, Margolis B. 2000. Molecular cloning and characterization of Pals, proteins associated with mLin-7. J Biol Chem 275: 11425-11431.

Karp CM, Tan TT, Mathew R, Nelson D, Mukherjee C, Degenhardt K, Karantza-Wadsworth V, White E. 2008. Role of the polarity determinant crumbs in suppressing mammalian epithelial tumor progression. Cancer Res 68: $4105-4115$.

Klose S, Flores-Benitez D, Riedel F, Knust E. 2013. Fosmidbased structure-function analysis reveals functionally distinct domains in the cytoplasmic domain of Drosophila crumbs. G3 (Bethesda) 3: 153-165.
Laprise P, Beronja S, Silva-Gagliardi NF, Pellikka M, Jensen AM, McGlade CJ, Tepass U. 2006. The FERM protein Yurt is a negative regulatory component of the Crumbs complex that controls epithelial polarity and apical membrane size. Dev Cell 11: 363-374.

Laprise P, Lau KM, Harris KP, Silva-Gagliardi NF, Paul SM, Beronja S, Beitel GJ, McGlade CJ, Tepass U. 2009. Yurt, Coracle, Neurexin IV and the $\mathrm{Na}^{+}, \mathrm{K}^{+}$-ATPase form a novel group of epithelial polarity proteins. Nature 459: $1141-1145$.

Lemmers C, Michel D, Lane-Guermonprez L, Delgrossi MH, Medina E, Arsanto JP, Le Bivic A. 2004. CRB3 binds directly to Par6 and regulates the morphogenesis of the tight junctions in mammalian epithelial cells. Mol Biol Cell 15: 1324-1333.

Letizia A, Sotillos S, Campuzano S, Llimargas M. 2011. Regulated Crb accumulation controls apical constriction and invagination in Drosophila tracheal cells. J Cell Sci 124: $240-251$.

Li Y, Wei Z, Yan Y, Wan Q, Du Q, Zhang M. 2014. Structure of Crumbs tail in complex with the PALS1 PDZ-SH3GK tandem reveals a highly specific assembly mechanism for the apical Crumbs complex. Proc Natl Acad Sci 111: 17444-17449.

Ling C, Zheng Y, Yin F, Yu J, Huang J, Hong Y, Wu S, Pan D. 2010. The apical transmembrane protein Crumbs functions as a tumor suppressor that regulates Hippo signaling by binding to Expanded. Proc Natl Acad Sci 107: 10532- 10537 .

Loie E, Charrier LE, Sollier K, Masson JY, Laprise P. 2015. CRB3A controls the morphology and cohesion of cancer cells through Ehm2/p114RhoGEF-dependent signaling. Mol Cell Biol 35: 3423-3435.

Lu H, Bilder D. 2005. Endocytic control of epithelial polarity and proliferation in Drosophila. Nat Cell Biol 7: 12321239.

Makarova O, Roh MH, Liu CJ, Laurinec S, Margolis B. 2003. Mammalian Crumbs3 is a small transmembrane protein linked to protein associated with Lin-7 (Pals1). Gene 302: 21-29.

Mao X, Li P, Ren Y, Li J, Zhou C, Yang J, Liu P. 2015. Cell polarity protein CRB3 is an independent favorable prognostic factor for clear cell renal cell carcinoma. Int J Oncol 46: $657-666$.

Medina E, Williams J, Klipfell E, Zarnescu D, Thomas G, Le Bivic A. 2002. Crumbs interacts with moesin and $\beta$ (Heavy)-spectrin in the apical membrane skeleton of Drosophila. J Cell Biol 158: 941-951.

Nelson WJ. 2009. Remodeling epithelial cell organization: Transitions between front-rear and apical-basal polarity. Cold Spring Harb Perspect Biol 1: a000513.

Nelson WJ, Dickinson DJ, Weis WI. 2013. Roles of cadherins and catenins in cell-cell adhesion and epithelial cell polarity. Prog Mol Biol Trans Sci 116: 3-23.

Pieczynski J, Margolis B. 2011. Protein complexes that control renal epithelial polarity. Am J Physiol Renal Physiol 300: F589-F601.

Ponuwei GA. 2016. A glimpse of the ERM proteins. J Biomed Sci 23: 35.

Robinson BS, Huang J, Hong Y, Moberg KH. 2010. Crumbs regulates Salvador/Warts/Hippo signaling in Drosophila 
via the FERM-domain protein Expanded. Curr Biol 20: $582-590$.

Roh MH, Makarova O, Liu CJ, Shin K, Lee S, Laurinec S, Goyal M, Wiggins R, Margolis B. 2002. The Maguk protein, Pals1, functions as an adapter, linking mammalian homologues of Crumbs and Discs Lost. J Cell Biol 157: $161-172$.

Roh MH, Fan S, Liu CJ, Margolis B. 2003. The Crumbs3Pals1 complex participates in the establishment of polarity in mammalian epithelial cells. J Cell Sci 116: 28952906.

Saotome I, Curto M, McClatchey AI. 2004. Ezrin is essential for epithelial organization and villus morphogenesis in the developing intestine. Dev Cell 6: 855-864.

Schluter MA, Pfarr CS, Pieczynski J, Whiteman EL, Hurd TW, Fan S, Liu CJ, Margolis B. 2009. Trafficking of Crumbs3 during cytokinesis is crucial for lumen formation. Mol Biol Cell 20: 4652-4663.

Shin K, Fogg VC, Margolis B. 2006. Tight junctions and cell polarity. Annu Rev Cell Dev Biol 22: 207-235.

Slavotinek A, Kaylor J, Pierce H, Cahr M, DeWard SJ, Schneidman-Duhovny D, Alsadah A, Salem F, Schmajuk G, Mehta L. 2015. CRB2 mutations produce a phenotype resembling congenital nephrosis, Finnish type, with cerebral ventriculomegaly and raised $\alpha$-fetoprotein. Am J Hum Genet 96: 162-169.

Sotillos S, Diaz-Meco MT, Caminero E, Moscat J, Campuzano S. 2004. DaPKC-dependent phosphorylation of Crumbs is required for epithelial cell polarity in Drosophila. J Cell Biol 166: 549-557.

Sun S, Irvine KD. 2016. Cellular organization and cytoskeletal regulation of the Hippo signaling network. Trends Cell Biol 26: 694-704.

Szymaniak AD, Mahoney JE, Cardoso WV, Varelas X. 2015. Crumbs3-mediated polarity directs airway epithelial cell fate through the hippo pathway effector Yap. Dev Cell 34: 283-296.

Tepass U. 2012. The apical polarity protein network in Drosophila epithelial cells: Regulation of polarity, junctions, morphogenesis, cell growth, and survival. Anпu Rev Cell Dev Biol 28: 655-685.

Tepass U, Theres C, Knust E. 1990. crumbs encodes an EGFlike protein expressed on apical membranes of Drosophila epithelial cells and required for organization of epithelia. Cell 61: 787-799.

Tilston-Lunel AM, Haley KE, Schlecht NF, Wang Y, Chatterton AL, Moleirinho S, Watson A, Hundal HS, Prystowsky MB, Gunn-Moore FJ, et al. 2016. Crumbs 3b promotes tight junctions in an ezrin-dependent manner in mammalian cells. J Mol Cell Biol 8: 439-455.

Torkko JM, Manninen A, Schuck S, Simons K. 2008. Depletion of apical transport proteins perturbs epithelial cyst formation and ciliogenesis. J Cell Sci 121: 1193-1203.

van den Hurk JA, Rashbass P, Roepman R, Davis J, Voesenek KE, Arends ML, Zonneveld MN, van Roekel MH, Cameron K, Rohrschneider K, et al. 2005. Characterization of the Crumbs homolog 2 (CRB2) gene and analysis of its role in retinitis pigmentosa and Leber congenital amaurosis. Mol Vis 11: 263-273.

van de Pavert SA, Kantardzhieva A, Malysheva A, Meuleman J, Versteeg I, Levelt C, Klooster J, Geiger S, Seeliger MW, Rashbass P, et al. 2004. Crumbs homologue 1 is required for maintenance of photoreceptor cell polarization and adhesion during light exposure. J Cell Sci 117: 41694177.

Varelas X, Samavarchi-Tehrani P, Narimatsu M, Weiss A, Cockburn K, Larsen BG, Rossant J, Wrana JL. 2010. The Crumbs complex couples cell density sensing to Hippo-dependent control of the TGF- $\beta-S M A D$ pathway. Dev Cell 19: 831-844.

Walton KD, Freddo AM, Wang S, Gumucio DL. 2016. Generation of intestinal surface: An absorbing tale. Development 143: 2261-2272.

Wei Z, Li Y, Ye F, Zhang M. 2015. Structural basis for the phosphorylation-regulated interaction between the cytoplasmic tail of cell polarity protein crumbs and the actinbinding protein moesin. J Biol Chem 290: 11384-11392.

Whiteman EL, Liu CJ, Fearon ER, Margolis B. 2008. The transcription factor snail represses Crumbs 3 expression and disrupts apico-basal polarity complexes. Oncogene 27: 3875-3879.

Whiteman EL, Fan S, Harder JL, Walton KD, Liu CJ, Soofi A, Fogg VC, Hershenson MB, Dressler GR, Deutsch GH, et al. 2014. Crumbs3 is essential for proper epithelial development and viability. Mol Cell Biol 34: 43-56.

Whitney DS, Peterson FC, Kittell AW, Egner JM, Prehoda KE, Volkman BF. 2016. Binding of Crumbs to the Par-6 CRIB-PDZ module is regulated by Cdc42. Biochemistry 55: $1455-1461$.

Xiao Z, Patrakka J, Nukui M, Chi L, Niu D, Betsholtz C, Pikkarainen T, Vainio S, Tryggvason K. 2011. Deficiency in Crumbs homolog 2 (Crb2) affects gastrulation and results in embryonic lethality in mice. Dev Dyn 240: 2646-2656.

Zanconato F, Cordenonsi M, Piccolo S. 2016. YAP/TAZ at the roots of cancer. Cancer Cell 29: 783-803. 


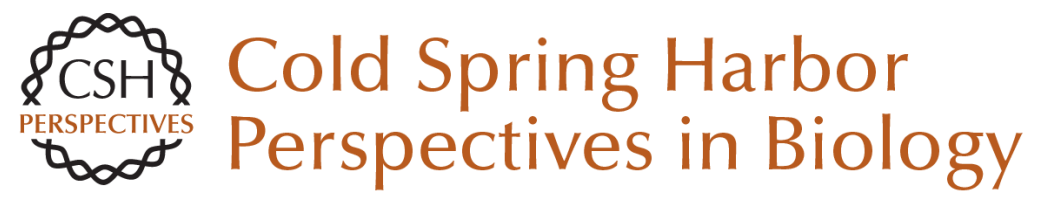

\section{The Crumbs3 Polarity Protein}

Ben Margolis

Cold Spring Harb Perspect Biol 2018; doi: 10.1101/cshperspect.a027961 originally published online March 13, 2017

\section{Subject Collection Cell Polarity}

Regulation of Cell Polarity by Exocyst-Mediated Trafficking Noemi Polgar and Ben Fogelgren

Phosphoinositides and Membrane Targeting in Cell Polarity Gerald R. Hammond and Yang Hong

Trafficking lon Transporters to the Apical Membrane of Polarized Intestinal Enterocytes Amy Christine Engevik and James R. Goldenring

Signaling Networks in Epithelial Tube Formation Ilenia Bernascone, Mariam Hachimi and Fernando Martin-Belmonte

Making Heads or Tails of It: Cell-Cell Adhesion in Cellular and Supracellular Polarity in Collective Migration Jan-Hendrik Venhuizen and Mirjam M. Zegers

Laminins in Epithelial Cell Polarization: Old Questions in Search of New Answers Karl S. Matlin, Satu-Marja Myllymäki and Aki Manninen

Epithelial Morphogenesis during Liver Development

Naoki Tanimizu and Toshihiro Mitaka

Targeting the Mucosal Barrier: How Pathogens Modulate the Cellular Polarity Network Travis R. Ruch and Joanne N. Engel
The Crumbs3 Polarity Protein Ben Margolis

Microtubule Motors in Establishment of Epithelial Cell Polarity Geri Kreitzer and Monn Monn Myat

Role of Polarity Proteins in the Generation and Organization of Apical Surface Protrusions Gerard Apodaca

Polarized Exocytosis Jingwen Zeng, Shanshan Feng, Bin Wu, et al.

Regulation of Transporters and Channels by Membrane-Trafficking Complexes in Epithelial Cells

Curtis T. Okamoto

Membrane Transport across Polarized Epithelia Maria Daniela Garcia-Castillo, Daniel J.-F. Chinnapen and Wayne I. Lencer

Mechanisms of Cell Polarity-Controlled Epithelial Homeostasis and Immunity in the Intestine Leon J. Klunder, Klaas Nico Faber, Gerard Dijkstra, et al.

The Biology of Ciliary Dynamics Kuo-Shun Hsu, Jen-Zen Chuang and Ching-Hwa Sung

For additional articles in this collection, see http://cshperspectives.cshlp.org/cgi/collection/

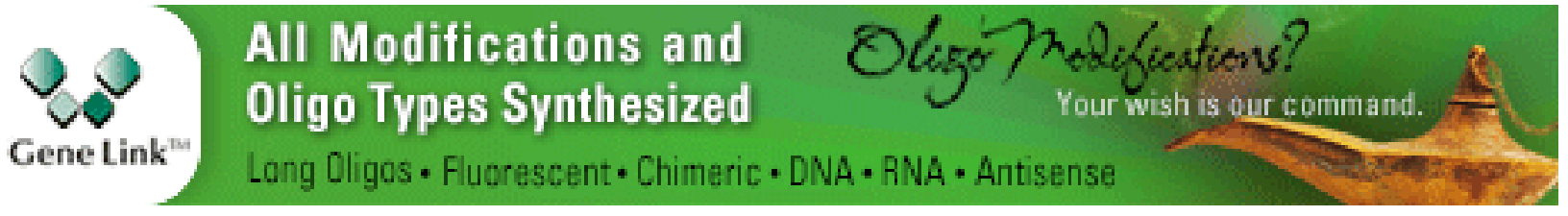

